\title{
A unique DNA-binding mode of African swine fever virus AP endonuclease
}

\author{
Yiqing Chen', Xi Chen', Qi Huang', Zhiwei Shao', Yanqing Gao', Yangyang Li', Chun Yang', Hehua Liu', Jixi Li (1)', \\ Qiyao Wang', Jinbiao Ma $\mathbb{D}^{1}$, Yong-Zhen Zhang ${ }^{3,4}$, Yijun $\mathrm{Gu}^{5}$ and Jianhua Gan ${ }^{1}$
}

\begin{abstract}
African swine fever virus (ASFV) is highly contagious and can cause lethal disease in pigs. ASFV is primarily replicated in the cytoplasm of pig macrophages, which is oxidative and caused constant damage to ASFV genome. ASFV AP endonuclease (AsfvAP) catalyzes DNA cleavage reaction at the abasic site and is a key enzyme of ASFV base excision repair (BER) system. Although it plays an essential role in ASFV survival in host cells, the basis underlying substrate binding and cleavage by AsfvAP remains unclear. Here, we reported the structural and functional studies of AsfvAP, showing that AsfvAP adopts a novel DNA-binding mode distinct from other APs. AsfvAP possesses many unique structural features, including one narrower nucleotide-binding pocket at the active site, the C16-C20 disulfide bondcontaining region, and histidine-rich loop. As indicated by our mutagenesis, in vitro binding and cleavage assays, these features are important for AsfvAP to suit the acidic and oxidative environment. Owing to their functional importance, these unique features could serve as targets for designing small molecule inhibitors that could disrupt the repair process of ASFV genome and help fight against this deadly virus in the future.
\end{abstract}

\section{Introduction}

African swine fever virus (ASFV) is a large doublestranded DNA (dsDNA) virus, and also the only member of the Asfarviridae family. ASFV is highly contagious ${ }^{1}$ and can cause lethal disease in pigs ${ }^{2}$. To date, ASFV has been found in many countries of the world and has caused a substantial loss in agricultural industry since its first report in Kenya in $1921^{3}$. To prevent a nationwide epidemic, more than 500,000 pigs were killed in Cuba in 1971, which was labeled the "most alarming event" of 1971 by the United Nations Food and Agricultural Organization $^{4}$. In 2011, the virus killed over 300,000 pigs in the Russian Federation region. Since the first report in

\footnotetext{
Correspondence: Yong-Zhen Zhang (zhangyongzhen@icdc.cn) or

Yijun Gu (guyijun@sari.ac.cn) or Jianhua Gan (ganjhh@fudan.edu.cn)

'State Key Laboratory of Genetic Engineering, Collaborative Innovation Center of Genetics and Development, Shanghai Public Health Clinical Center, School of Life Sciences, Fudan University, 200438 Shanghai, China

${ }^{2}$ State Key Laboratory of Bioreactor Engineering, East China University of

Science and Technology, 200237 Shanghai, China

Full list of author information is available at the end of the article

These authors contributed equally: Yiqing Chen, Xi Chen
}

August $2018^{5}$, ASFV has been reported in over 30 provinces in China, caused huge economic losses, as well as immediate pork shortage. Due to its serious threat to the agricultural industry, ASFV has attracted tremendous attention from governments and scientists in the past decade in the world. Unfortunately, no vaccine or other useful treatment against this virus has been developed so far ${ }^{6}$.

ASFV is one of the most complex viruses known to date. Its genome varies between 170 and $190 \mathrm{~kb}$, encoding more than 150 proteins that are involved in various stages of ASFV life cycle, including suppression of host immune response, entry into host cells, gene expression, and virion assembly ${ }^{7}$. ASFV is primarily replicated in the cytoplasm of swine macrophage cells ${ }^{8}$. The cytoplasm of macrophages is very rich in free oxygen radicals that caused constant damages to ASFV genome ${ }^{9,10}$. To efficiently overcome these damages, especially for DNA abasic sites (AP sites), ASFV evolved its own base excision repair (BER) system, including an AP endonuclease (AsfvAP), a repair polymerase (AsfvPolX), and a ligase (AsfvLIG). 
Unlike other repair enzymes, the fidelities of both AsfvPolX ${ }^{11}$ and $A s f v$ LIG $^{12}$ are very low. Therefore, in addition to maintaining the integrity of viral genome, these enzymes may also play important roles in strategic mutagenesis ${ }^{11}$ and genotype formation of ASFV, which further complicated the epidemiology, diagnosis, and prevention of $\mathrm{ASFV}^{13-15}$.

Owing to their functional importance, ASFV repair enzymes have been extensively studied ${ }^{16,17}$. The NMR structures of AsfuPolX were independently reported by Tsai and Mullen previously ${ }^{18-20}$. The crystal structures of both $A s f v \mathrm{PolX}^{21}$ and $A s f v \mathrm{LIG}^{22}$ were determined by our group very recently. In addition to the intrinsic mechanism of DNA repair, these structures also unraveled many features unique to ASFV repair enzymes, such as the $5^{\prime}$ phosphate group $\left(5^{\prime}-\mathrm{P}\right)$ binding pocket located at the finger domain of AsfvPolX and the DNA-binding domain located at the N-terminus of AsfvLIG.

AsfvAP belongs to the class II AP endonuclease family; it mainly catalyzes DNA cleavage reaction at the $5^{\prime}$ side of abasic site (AP), generating $3^{\prime}-\mathrm{OH}$ group and $5^{\prime}$-deoxyribose phosphate $(\mathrm{dRP})^{23}$. AsfvAP-catalyzed reaction represents one key step in ASFV BER pathway. Deletion of AsfvAP-coding gene (pE296R) lowered the growth rate of the virus to $2-4 \%$ of the parental virus in swine macrophages ${ }^{17}$. AsfvAP shares very low sequence identity $(\sim 10 \%)$ with the homologous proteins including E. Coli Nfo (EcNfo), G. Kaustophilus Nfo (GkNfo), B. Anthracis Nfo (BaNfo), T. Thermus Nfo (TtNfo), and M. tuberculosis EndoIV (MtEndoIV) (Fig. 1a). Similar to $E c N f$, AsfvAP possesses $3^{\prime} \rightarrow 5^{\prime}$ exonuclease, $3^{\prime}$-phosphodiesterase, $3^{\prime}$-phosphase and weak nucleotide incision repair (NIR) activities. However, compared with EcNfo and other homologous proteins, AsfvAP is more specific for the abasic sites ${ }^{24,25}$. DNA binding and cleavage activities of AsfvAP are very sensitive to the redox environment ${ }^{25}$. Due to the lack of structural data, the overall folding, DNA recognition, and the basis for redox-sensing of AsfvAP remain unclear. Herein we report two complex structures of $A s f v$ AP (AsfvAP/DNA-1 and AsfvAP/DNA2, Supplementary Table S1), showing that AsfuAP adopts one novel DNA-binding mode. Via mutagenesis and in vitro binding and cleavage assays, we also explored the functional roles of the unique features of AsfvAP. Our studies not only advance our understanding on ASFV BER pathway but also provide potential basis for anti-ASFV drug designing.

\section{Results}

\section{$\mathrm{pH}$-dependence of AP endonuclease activity}

Normally, macrophage cells acidify their cytoplasm environment to clear intruders ${ }^{26}$. To test the potential effects of acidification on the catalytic efficiency of $A s f$ $v$ AP, we designed one FAM-labeled DNA (DNA-3: 5'-
GGTAAGGGCAGCGTCCFCGACGAGGAATGCA-FAM3', 3'-CCATTCCCGTCGCAGGGGCTGCTCCTTACGT$5^{\prime}$, the abasic site was shown as capital letter $F$ in the sequence) and performed in vitro cleavage assays. As depicted in Fig. 1b-d and Supplementary Fig. S1, wild type (WT) AsfuAP could cleave DNA-3 within a wide range of $\mathrm{pH}$. The highest activity was observed under $\mathrm{pH} 6.3$, and the apparent rate constant $\left(K_{\text {obs }}\right)$ was about $0.55 \times 10^{-3} \mathrm{~min}^{-1}$. Increasing the buffer $\mathrm{pH}$ to 7.5 or decreasing it to 5.0 caused 5-7-fold reduction in the catalytic efficiency of AsfvAP, but the protein retained $40 \%$ activity when the $\mathrm{pH}$ varied between 5.9 and 7.0 (Supplementary Table S2).

The above observations suggested that AsfuAP prefers weak acidic condition for its AP endonuclease activity. The sequence identities between AsfvAP and the homologous proteins are very low (Fig. 1a). For direct comparison, we purified $E c \mathrm{Nfo}$ and performed in vitro cleavage assays. As depicted in Fig. 1e-g and Supplementary Fig. S2, EcNfo could cleave DNA-3 within a wide range of $\mathrm{pH}$. The $\mathrm{K}_{\mathrm{obs}}$ value of $E c \mathrm{Nfo}$ was around $0.20 \times$ $10^{-3} \mathrm{~min}^{-1}$ under $\mathrm{pH} 5.0-6.5$, and increased to $0.49 \times$ $10^{-3} \mathrm{~min}^{-1}$ and $0.56 \times 10^{-3} \mathrm{~min}^{-1}$ at $\mathrm{pH} 7.5$ and 8.0 (Supplementary Table S3), respectively. Different from $A s f v A P$, the cleavage assay results suggested that $E c \mathrm{Nfo}$ prefers basic condition for its AP endonuclease activity. In addition to the main abasic site cleavage products, some minor products were also produced by $E c \mathrm{Nfo}$ under $\mathrm{pH}$ 7.0-8.0 (Fig. $1 \mathrm{~g}$ and Supplementary Fig. S2). These minor products should correspond to $3^{\prime}$-FAM-A, which were generated by the $3^{\prime} \rightarrow 5^{\prime}$ exonuclease activity of $E c \mathrm{Nfo}$. Unlike $E c$ Nfo, AsfvAP did not show obvious $3^{\prime} \rightarrow 5^{\prime}$ exonuclease activity under all the tested conditions (Supplementary Fig. S1). Taken together, these data suggested that AsfvAP has higher abasic site selectivity.

\section{Overall folding of the AsfvAP/DNA-1 complex}

To unravel the molecular basis underlying substrate binding and cleavage by AsfvAP, we performed crystallographic studies and solved two complex structures of AsfvAP, AsfvAP/DNA-1, and AsfvAP/DNA-2. Both DNA1 (5'-GCAGCGTCCFCGACGAGG-3' and 3'-CGTCGCA GGGGCTGCTCC-5') and DNA-2 (5'-GCAGCGTCAC CGACGAAA-3' and 3'-CGTCGCAGGGGCTGCTC C-5') are 18-bp dsDNAs, containing one abasic site and one A:G mispair in the middle, respectively. AsfvAP/ DNA-1 crystal belongs to $\mathrm{P} 2{ }_{1}$ space group. Per asymmetric unit contains two AsfvAP/DNA-1 complexes, and each complex is formed by one AsfvAP protein and one DNA-1 molecule (Fig. 2a). AsfvAP is of $\alpha / \beta$ fold in nature; the $\beta$-strands form one $\beta$-barrel in the center, surrounded by $\alpha$-helices at the outer side. DNA molecule adopts regular B-form-like conformations at both ends, but it was severely bent with the angle between the two helical axes being about $100^{\circ}$ in the middle. 


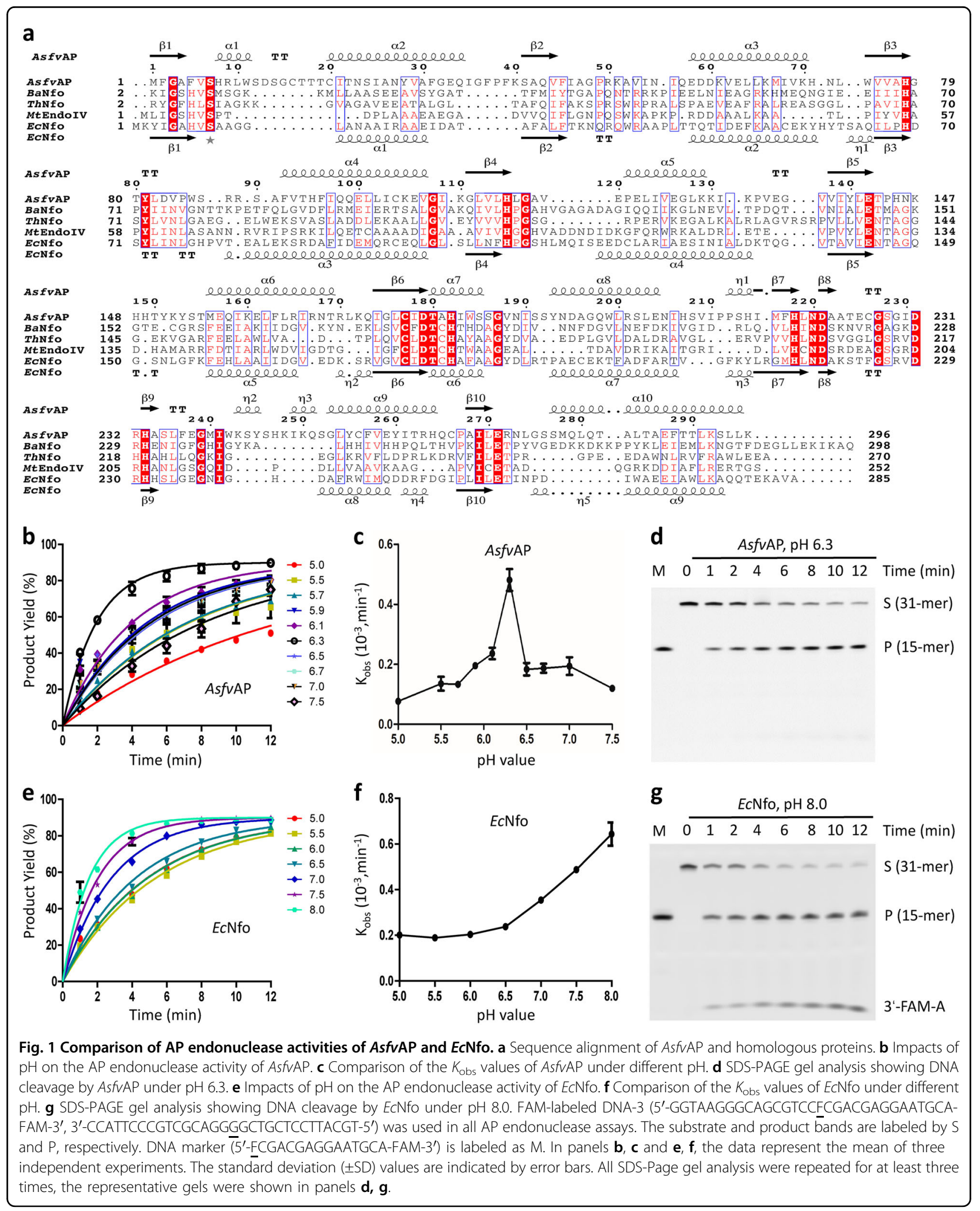

Intact DNA-1 duplex was utilized in the crystallization process. However, as confirmed by the clear $2 \mathrm{~F}_{\mathrm{o}}-\mathrm{F}_{\mathrm{c}}$ electron density maps, the DNA was cleaved at the $5^{\prime}$ side of the abasic site in the structure (Fig. 2b). Three welldefined $\mathrm{Zn}^{2+}$ ions were captured in the structure, coordinating with the side chains of His78, His115, Glu142, 

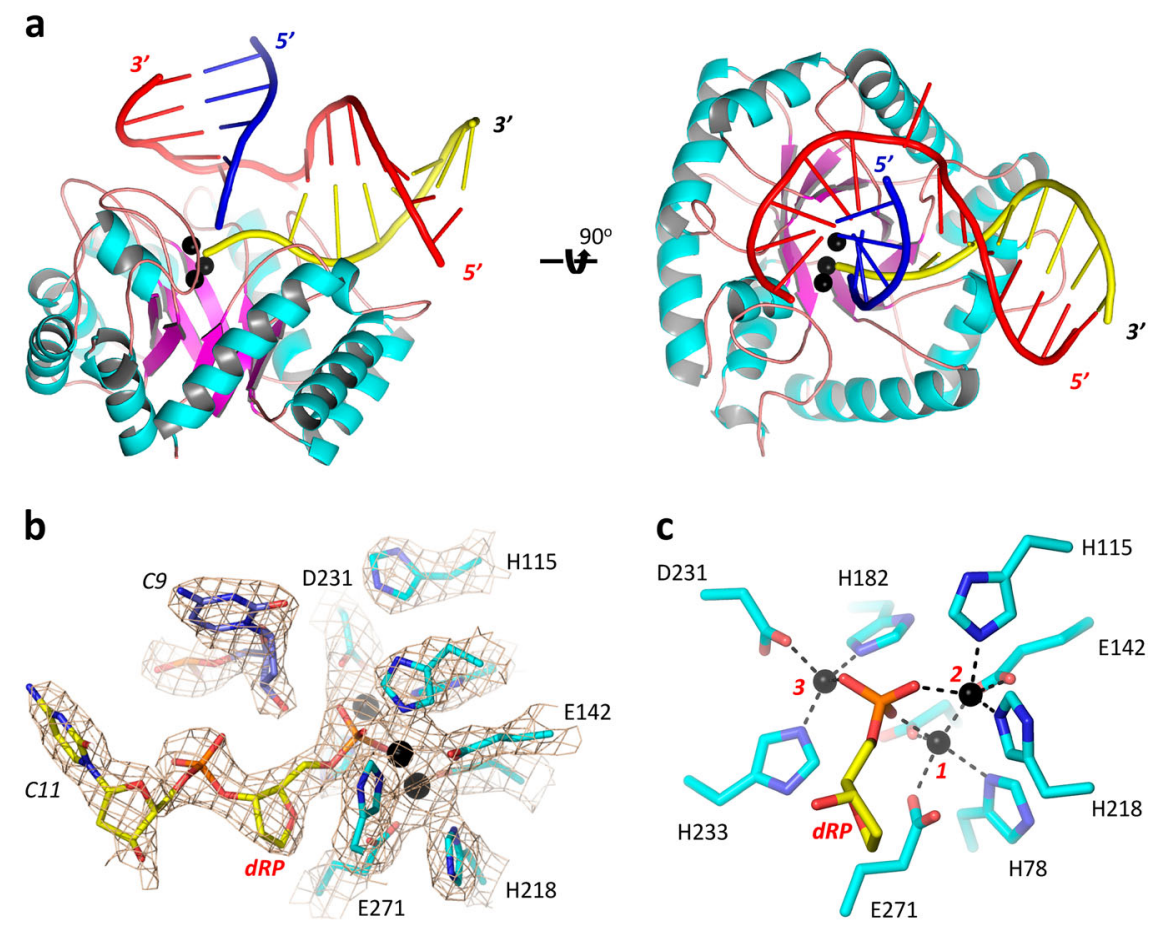

Fig. 2 Structure of AsfvAP/DNA-1 complex. a Cartoon view showing the overall fold of AsfvAP/DNA-1 complex. a-helices, $\beta$-strands and loops of AsfvAP are colored in cyan, purple, and pink, respectively. The uncleaved strand, the upstream and downstream of the product strand of DNA-1 are colored in red, blue and yellow, respectively. $\mathbf{b}$ Conformations of the active site residues and nucleotides. $2 \mathrm{~F}_{\mathrm{o}}-\mathrm{F}_{\mathrm{c}}$ electron density map was contoured at 1.5 sigma level. c Detailed coordination of the $\mathrm{Zn}^{2+}$ ions. The $\mathrm{Zn}^{2+}$ ions are shown as spheres in black in all panels.

Asp179, His182, Asp231, His233, and Glu271. In addition, the $\mathrm{Zn}^{2+}$ ions also forms direct coordination with the $5^{\prime}$ phosphate group of $\mathrm{dRP}$, the product of the abasic site cleavage (Fig. 2c). Taken together, these observations demonstrated the catalytic activity of AsfvAP and indicated that the AsfvAP/DNA-1 complex was assembled in a catalytic form.

\section{Molecular basis for target DNA recognition by AsfvAP}

The AsfvAP/DNA-1 complex structure was refined at atomic resolution $(2.35 \AA)$, which revealed the detailed basis for target DNA recognition by AsfvAP. The DNA molecule mainly interacts with AsfvAP in three regions: R1, R2, and R3 (Fig. 3a). The R1 region $\left({ }_{8}\right.$ HRLWSDSGCTTTC $\left._{20}\right)$ is composed of one short $\alpha$ turn immediately followed by a loop. The $\mathrm{R} 2$ region is one histidine-rich loop $\left({ }_{145} \mathrm{HNKHHT}_{150}\right)$, connecting $\beta 5$ strand and $\alpha 6$ helix at the central region. The R3 region $\left({ }_{272} \mathrm{RNLGSSMQLQ}_{281}\right)$ consists of the $\beta 10-\alpha 10$ linker and the $\mathrm{N}$-terminal half of $\alpha 10$ helix at the $\mathrm{C}$ terminus. R1 and R3 are close to each other, whereas R2 is isolated in space.

The conformation of the target DNA was stabilized by several different types of interactions. The R1 region (Fig. 3b) mainly interacts with DNA through hydrogen binding (H-bond). The NE2 atom of His8 (the first residue of R1) forms one $\mathrm{H}$-bond with the phosphate group of A13 of the product strand; A13 is 3-nt downstream of dRP. Ser14 is located at the middle of $\mathrm{R} 1$, and also forms one direct $\mathrm{H}$-bond with DNA via its side chain. However, instead of the product strand, it interacts with $\mathrm{C} 2$ near the $5^{\prime}$-end of the uncleaved strand.

Of the six residues of the R2 loop, three are histidines, including His145, His148, and His149. Both His148 and His149 locate at the tip region of the loop and directly interact with the uncleaved DNA strand (Fig. 3c). Via its main chain $\mathrm{N}$ atom and the side chain NE2 atom, His148 forms two H-bonds: one with the OP1 atom of $\mathrm{C} 13$ and another with the OP2 atom of G14. Obviously, the NE2 atom of His149 forms one H-bond with the OP2 atom of C13. His145 resides at the $\mathrm{N}$-terminus of the $\mathrm{R} 2$ loop. Although it does not form direct $\mathrm{H}$-bond interaction with the DNA, it interacts with the $5^{\prime}$-phosphate group of dRP through one water molecule (Fig. 3d).

The side chain of Asn273 of R3 points toward the product strand (Fig. 3e). The ND2 atom of Asn273 forms one $\mathrm{H}$-bond with the $\mathrm{O}^{\prime}$ ' atom of dRP, stabilizing the dRP conformation from the $3^{\prime}$ side. The conformation of $\mathrm{C} 11$, located at the right downstream of $\mathrm{dRP}$, was stabilized by two types of interactions: the OP2 atom of C11 interacts with hydroxyl group of the side chain of Tyr81 (Fig. 3e) and the nucleobase of C11 stacks against the guanidine 


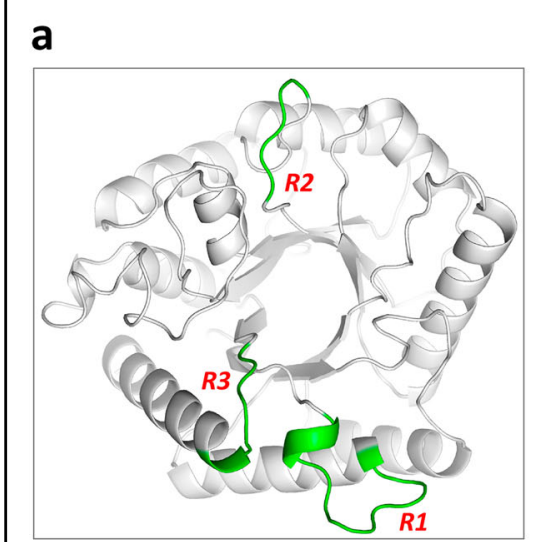

d

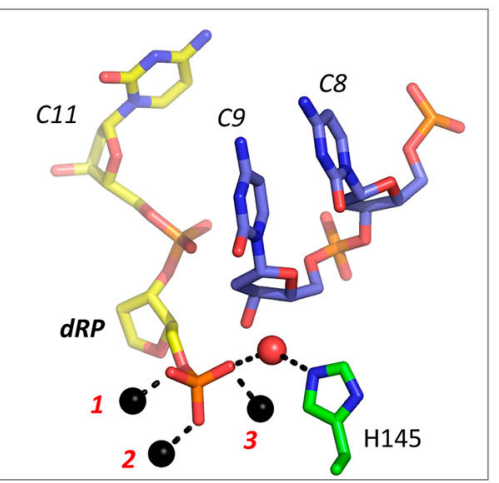

b

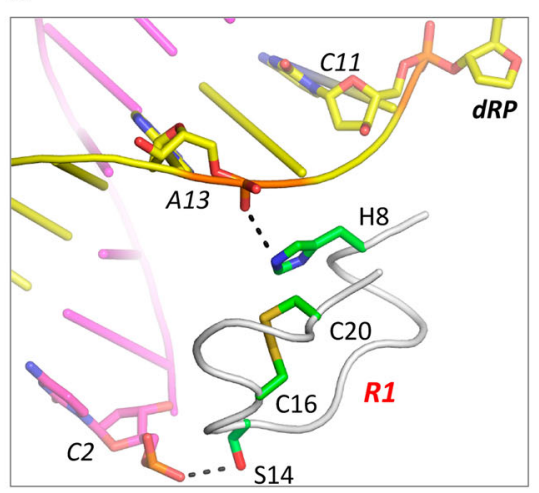

e

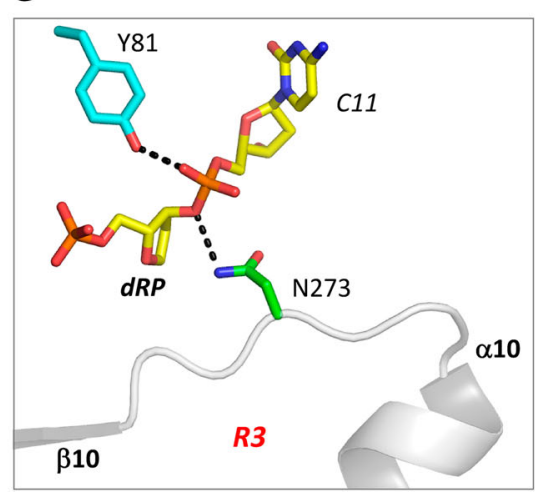

C

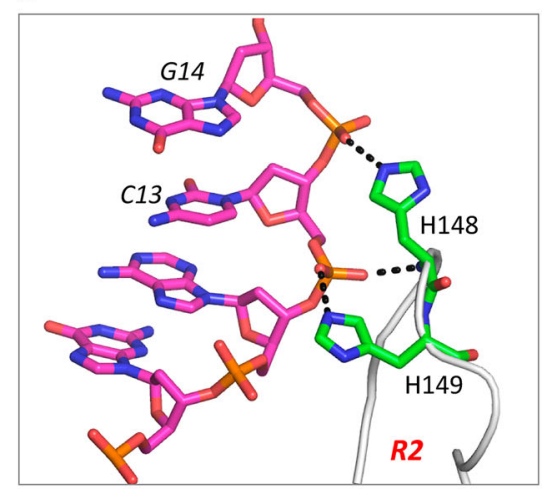

f

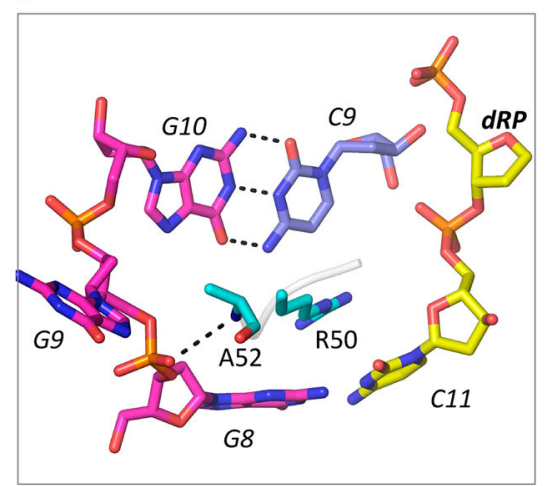

Fig. 3 Protein-DNA interactions observed in the AsfvAP/DNA-1 complex. a Cartoon view showing the conformations and relative orientations of the R1, R2 and R3 regions, which are colored in green. $\mathbf{b}$ Detailed interactions between DNA-1 and the R1 region. DNA-1 and R1 are shown as cartoon. The interacting residues (His8 and Ser14) and nucleotides (A13 and C2) are highlighted by sticks. dRP nucleotide of DNA-1 and Cys16-Cys20 disulfide bond of R1 are also highlighted by sticks. $\mathbf{c}-\mathbf{d}$ Detailed interactions between DNA-1 and the R2 region. $\mathrm{Zn}^{2+}$ ions and water molecule are shown as spheres in black and red, respectively. e Detailed interactions between DNA-1 and the R3 region. Besides Asn273 of the R3 region, the conserved Tyr81 residue is also highlighted by sticks. $\mathbf{f}$ Interactions between nick region nucleotides and AsfvAP residues.

group of Arg50 (Fig. 3f). The side chain of Arg50 is deeply inserted into the minor grove of the duplex, which is most likely the major factor for the significant bending of the DNA. The phosphate group of G9 of the uncleaved strand forms one $\mathrm{H}$-bond with the main chain $\mathrm{N}$ atom of Ala52. However, due to lacking of pairing with other nucleotide, the nucleobase of G9 was flipped out.

\section{A novel DNA-binding mode of AsfvAP}

Comparison with the reported class II AP endonuclease structures showed that AsfvAP has similar secondary structure topology as $B a \mathrm{Nfo}, M t \mathrm{EndoIV}^{27}$, and $T t \mathrm{Nfo}^{28}$, in which the central $\beta$-barrels can superimpose with each other very well (Fig. 4a and Supplementary Fig. S3). However, the orientations of the outer $\alpha$-helices of AsfvAP are significantly different from those of $B a \mathrm{Nfo}, M t \mathrm{En}$ $\mathrm{doIV}$, and $T t \mathrm{Nfo}$; the root mean square deviation (rmsd) values between AsfvAP and the three homologous structures are all greater than $4.0 \AA$. In addition to apo-structure, several complex structures of $E c \mathrm{Nfo}$ have also been reported previously ${ }^{29,30}$. Compared to AsfvAP, the overall conformation of $E c \mathrm{Nfo}$ is more similar to those of $B a \mathrm{Nfo}$, $M t$ EndoIV, and $T t \mathrm{Nfo}$, might due to the higher sequence identities between them (Fig. 1a). The rmsd value between substrate-bound $E c$ Nfo (PDB: $2 \mathrm{NQJ}$ ) and $T t \mathrm{Nfo}$ is $1.7 \AA$ and only $1.3 \AA$ between $E c$ Nfo and $B a \mathrm{Nfo}$, suggesting that these proteins may share similar mode in DNA binding. AsfvAP mainly binds DNA at the R1, R2, and R3 regions (Fig. 3). However, as depicted in Fig. 4a, b, the conformations of R1, R2, and R3 in AsfvAP are significantly different from those of the homologous proteins.

Besides the conformational difference between the proteins, structural superposition also revealed significant conformational differences of the DNAs (Fig. $4 c)$. In the $E c \mathrm{Nfo} /$ substrate complex structure, DNA forms several stable $\mathrm{H}$-bond interactions with $E c \mathrm{Nfo}$. However, instead of the side chains, these interactions are mainly mediated by the main chains of $E c N f o$. Compared with the DNA in the $E c \mathrm{Nfo} /$ substrate complex, the DNA in AsfvAP/DNA-1 structure is rotated 

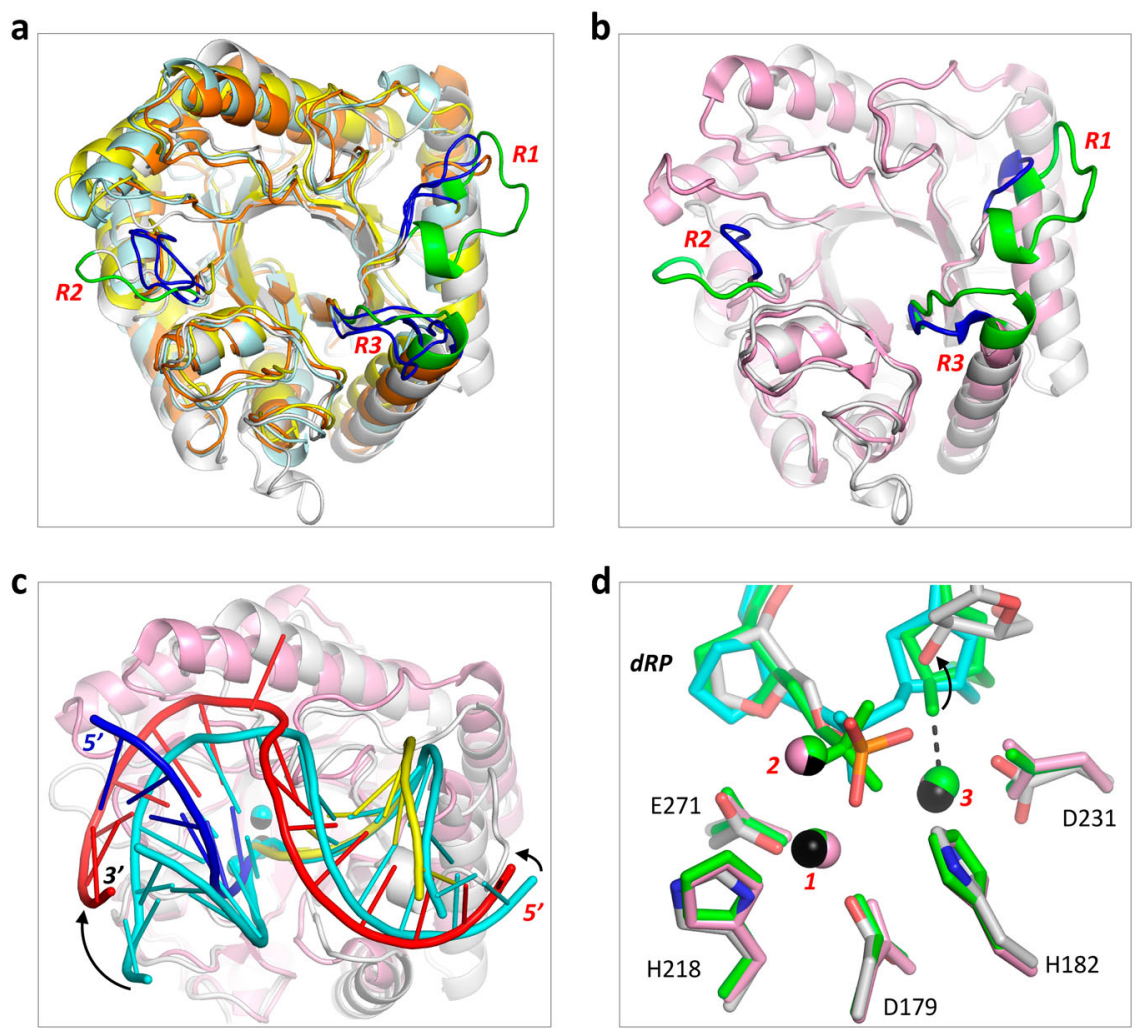

Fig. 4 Structural comparison between AsfvAP and homologous proteins. a Superposition of AsfvAP, BaNfo, MtEndolV, and TtNfo structures. b, c Superposition of AsfvAP/DNA-1 and ECNfo/substrate (PDB: 2NQJ) complexes. d Superposition of the DNAs bound in the active sites of AsfvAP/DNA-1, EcNfo/product (PDB: 2NQ9), and EcNfo/substrate complexes. In panels a, b, AsfvAP, BaNfo, MtEndolV, TtNfo, and EcNfo are colored in white, cyan, yellow, orange, and pink, respectively. The R1-R3 regions are colored in green for AsfvAP, whereas they are colored in blue for all other proteins. In panel c, AsfvAP and ECNfo are colored in white and pink, respectively. For the AsfvAP/DNA-1 complex, the uncleaved strand, upstream and downstream of the cleaved strand are colored in red, blue and yellow, respectively. $\mathrm{Zn}^{2+}$ ions are shown as black spheres. For the EcNfo/substrate complex, DNA strands and $\mathrm{Zn}^{2+}$ ions are all colored in cyan. In panel $\mathbf{d}$, DNA, protein, and $\mathrm{Zn}^{2+}$ ions are colored in atomic colors $(\mathrm{C}$, white; $\mathrm{N}$, blue; $\mathrm{O}$, red; $\mathrm{P}$, orange; $\mathrm{Zn}^{2+}$, black) for the AsfvAP/DNA-1 complex. The EcNfo/product complex is colored in green. For the EcNfo/substrate complex, DNA is colored in cyan, protein and $\mathrm{Zn}^{2+}$ ions are colored in pink.

about $10^{\circ}$ anti-clockwise and $30^{\circ}$ clockwise toward the $5^{\prime}$ and $3^{\prime}$ ends of the uncleaved strand, respectively. The cleavage site dRPs adopt similar conformations in Asf$v \mathrm{AP} / \mathrm{DNA}-1, E c \mathrm{Nfo} /$ product (PDB: 2NQ9), and $E c \mathrm{Nfo} /$ substrate complexes (Fig. $4 \mathrm{~d}$ ). Like the $E c \mathrm{Nfo} /$ substrate complex, the $3^{\prime}$-hydroxyl group did not leave the active site and coordinated with the catalytic $\mathrm{Zn}^{2+}$ ion in the $E c \mathrm{Nfo} /$ product complex, representing a product-bound state immediately after the cleavage reaction. Compared to the product and substrate complexes of $E c \mathrm{Nfo}$, the $3^{\prime}$-hydroxyl group was shifted away by $\sim 1.7 \AA$ in the AsfvAP/DNA-1 complex, which may represent one product-releasing state. The conformations of the catalytic $\mathrm{Zn}^{2+}$ ions and the coordinating residues are almost identical in the three complex structures (Fig. 4d). Taken together, these observations suggested that AsfvAP adopts a novel DNA-binding mode, but shares the same catalytic mechanism with other class II AP endonucleases.

\section{R1 and R2 regions are important for AsfvAP function}

As described above, AsfvAP adopts a novel DNAbinding mode and the majorities of the DNA-interacting residues are unique to AsfvAP (Figs. 1a and 3). To verify the functional importance of these residues, we constructed several AsfvAP mutants and performed in vitro DNA binding and cleavage assays (Fig. $5 \mathrm{a}-\mathrm{c}$ and Supplementary Table S4). WT AsfuAP has strong DNA binding affinity, indicated by the low dissociation value $\left(K_{\mathrm{d}}, 0.81 \pm\right.$ $0.14 \mu \mathrm{M})$. Compared to WT AsfvAP, substitution of either His8 or Ser14 with Ala lowered the DNA binding affinity of the protein by $6 \sim 8$-folds. H8A/S14A double mutation led to more than 12 -fold reduction in DNA binding. In consistent with their weaker DNA binding affinities, the DNA-3 cleavage activities of H8A, S14A, and H8A/S14A mutants are all weaker than that of WT AsfvAP by 2-folds.

Compared to the homologous proteins, the $\mathrm{R} 1$ region (aa 8-20) of AsfvAP is significantly longer. In $E c \mathrm{Nfo}$, the $\mathrm{R} 1$ region is substituted by one 4 -amino-acid ( $\left.{ }_{10} \mathrm{AAGG}_{13}\right)$ 


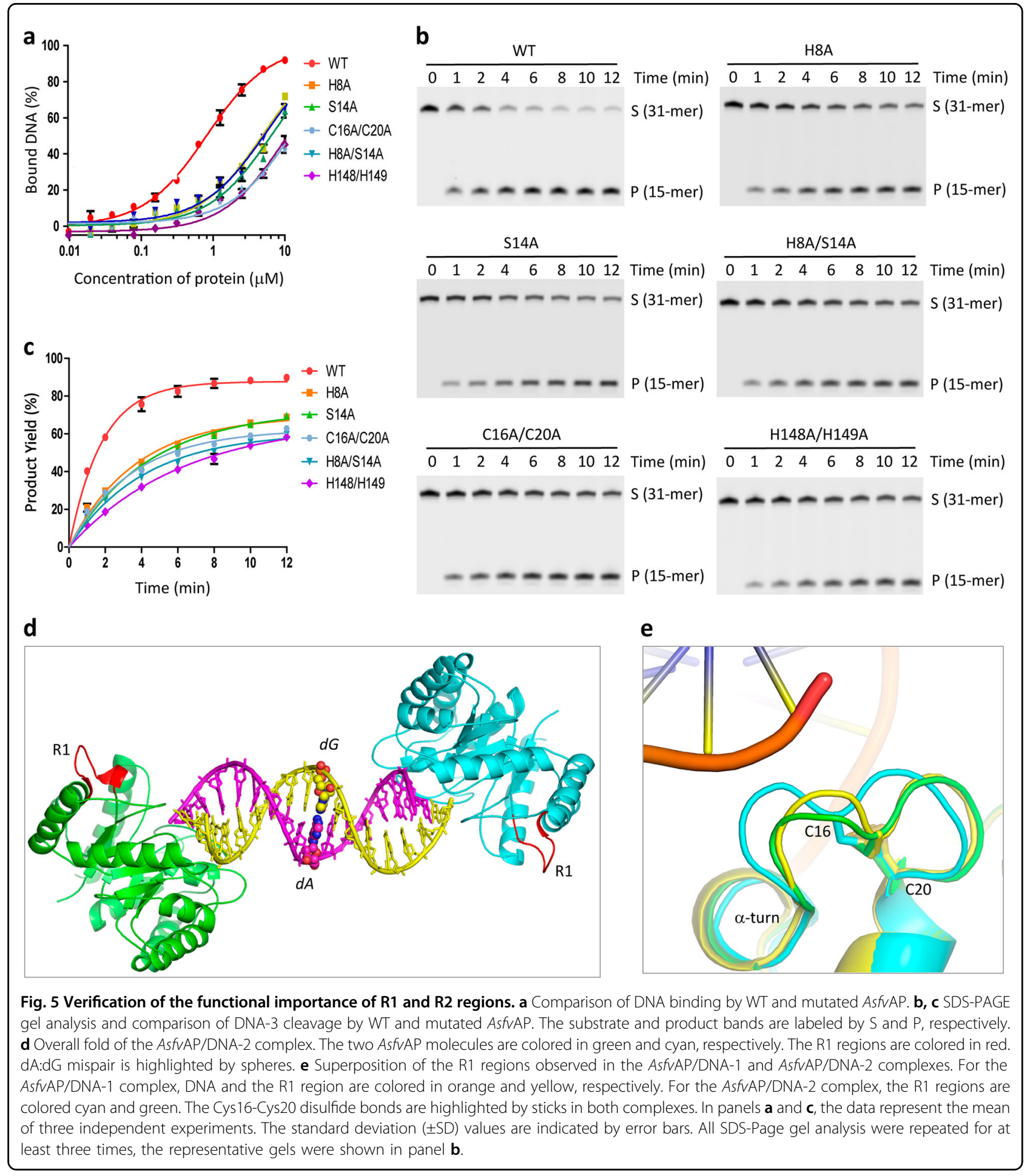

loop, which adopts one different conformation (Fig. 4b). In addition to single-point mutants, we also constructed one AsfvAP mutant (AsfvAP_Ec-loop) with the R1 region replaced by AAGG. As depicted in Supplementary Fig. S4, the loop replacement only caused 2-fold reduction in the protein's DNA binding affinity. However, it weakened the
DNA cleavage activity of the protein by 12 -folds (Supplementary Table S4). This observation indicated that the R1 region of AsfvAP could not be replaced by the corresponding loop of $E c$ Nfo.

AsfvAP contains 7 cysteine residues, including Cys16, Cys20, Cys104, Cys177, Cys226, Cys256, and Cys266. 
Previous studies suggested that AsfvAP forms one intramolecular $\mathrm{S}-\mathrm{S}$ bond ${ }^{25}$. Reducing reagents, such as dithiothreitol (DTT) and $\beta$-mercaptoethanol ( $\beta$-ME), can inhibit DNA binding and cleavage by AsfvAP. The inhibitory effects of DTT and $\beta$-ME can be reversed by $\mathrm{H}_{2} \mathrm{O}_{2}$, which is oxidative. As revealed by our AsfvAP/DNA-1 complex, the S-S bond was formed between Cys16 and Cys20 within the R1 region. Surprisingly, although the $\mathrm{S}-\mathrm{S}$ bond is important for AsfvAP function, neither Cys16 nor Cys20 interacted with the DNA in the structure (Fig. 3). Puzzled by these observations, we performed thoroughly structural comparison and mutagenesis studies. In the AsfvAP/DNA2 structure, each DNA was bound by two AsfvAP molecules, but the DNA did not interact with any R1 residues (Fig. 5d). Cys16 could undergo subtle conformational changes, whereas the conformation of the neighboring $\alpha$ turn and Cys20 of R1 were well fixed in AsfuAP/DNA-1 and AsfvAP/DNA-2 complexes (Fig. 5e). The DNA binding and cleavage activities of $\mathrm{C} 16 \mathrm{~A} / \mathrm{C} 20 \mathrm{~A}$ double mutant are approximately 6-fold and 2-fold weaker than those of WT AsfvAP (Fig. 5a-c and Supplementary Table S4). The cytoplasm of macrophages is very rich in free oxygen radicals, which play a similar role as $\mathrm{H}_{2} \mathrm{O}_{2}$ in favoring S-S bond formation. We believed that the S-S bond of AsfvAP is evolved to help the virus to adapt the oxidative condition of macrophages.

The lengths of R2 are comparable in AsfvAP and the homologous proteins. However, the sequence of AsfvAP $\mathrm{R} 2$ is very different from those of the homologous proteins (Fig. 1a). In AsfvAP/DNA-1 complex, the R2 residues His148 and His149 form direct H-bond interactions with DNA-1 (Fig. 3c). To investigate the potential roles of the histidine residues, one $\mathrm{H} 148 \mathrm{~A} / \mathrm{H} 149 \mathrm{~A}$ double mutant was constructed and its DNA binding affinity and enzymatic activity were measured. Compared to WT AsfvAP, the DNA binding and cleavage activities of H148A/ H149A mutant are approximately 15-fold and 3.5-fold weaker (Fig. 5a-c and Supplementary Table S4). Like His148 and His149, His8, and His145 are also involved in DNA binding (Fig. 3). It is well known that histidine is quite different from other residues, and that its protonation state is very sensitive to the $\mathrm{pH}$ value of the environment. We speculated that these histidine residues are evolved to enhance the catalytic efficiency of AsfvAP (Fig. $1 \mathrm{~b}, \mathrm{c})$ and to help the virus survive under the acidic condition of macrophages.

\section{One narrower nucleotide-binding pocket in AsfvAP}

The R3 region of AsfvAP is 2-amino-acid shorter than that of $B a \mathrm{Nfo}$, but significantly longer than those of $M t$ EndoIV, TtNfo and EcNfo (Fig. 1a). Besides Asn273, which forms direct $\mathrm{H}$-bond interaction with $\mathrm{dRP}$ in the AsfvAP/DNA-1 complex (Fig. 3e), the neighboring Arg272 residue is also unique to AsfvAP. The side chain of
Arg272 forms four H-bond interactions with the surrounding residues, including one with the main chain of Leu219, one with the side chain of Asn220, and two with the side chain of Glu287 (Fig. 6a). Although Asn220 is highly conserved, Arg272 of AsfvAP is replaced by one Thr residue in $E c$ Nfo and other class II AP endonucleases (Fig. 1a). Unlike Arg, the side chain of Thr is too short to form direct $\mathrm{H}$-bond interaction with the conserved Asn residue. Compared to Arg272 in the AsfvAP/DNA-1 complex, the backbone of the corresponding Thr262 is shifted $3.0 \AA$ away from the dRP in the $E c \mathrm{Nfo} /$ substrate structure. In the homologous protein structures, the R3 region forms one loop (Fig. 4a, b and Supplementary Fig. S3). Asn273 in AsfvAP is substituted by residues Pro, Pro, Ala, and Ile in $B a \mathrm{Nfo}, T t \mathrm{Nfo} M t$ EndoIV and $E c \mathrm{Nfo}$, which are all hydrophobic and incapable of forming $\mathrm{H}$-bond interaction with DNA. As revealed by structural superposition, the backbone of Ile263 of $E c$ Nfo is shifted more than $4.0 \AA$ away from the corresponding Asn273 of Asf$v$ AP (Fig. 6b).

The dRP nucleotides adopt similar conformations in AsfvAP/DNA-1 and the $E c$ Nfo/substrate complex structures (Figs. $4 \mathrm{~d}$ and $6 \mathrm{~b}$ ). The sugar pucker of dRP was bound in one shallow pocket, and the bottom of the pocket is formed by the side chains of Phe5, Phe45, and Glu271 in AsfvAP. For EcNfo, the bottom of the dRPbinding pocket is formed by His7, Phe32, and Glu261. The gate to the pocket is formed by the side chains of Tyr81 and Asn273 in AsfuAP (Fig. 6c). Tyr81 is highly conserved (Fig. 1a) and adopts similar conformation with the corresponding Tyr72 in the EcNfo/substrate complex (Supplementary Fig. S5). However, due to the different conformation of the R3 region residues, especially Asn 273 in AsfvAP and Ile263 in $E c$ Nfo, the nucleotide-binding pocket of AsfvAP is much narrower than that of $E c$ Nfo.

To test the functional importance of the gating residues, we constructed two AsfvAP mutants (Y81A and N273A) and performed in vitro DNA binding assays (Fig. 6d). Surprisingly, the DNA binding affinities of Y81A mutant $(1.39 \pm 0.05 \mu \mathrm{M})$ and $\mathrm{N} 273 \mathrm{~A}$ mutant $(1.49 \pm 0.06 \mu \mathrm{M})$ were only slightly weaker than that of WT AsfvAP (Supplementary Table S4). Puzzled by these observations, we performed in vitro cleavage assays (Fig. 6e, f) and found that the DNA cleavage activities of Y81A and N273A mutants are about 240-fold and 35-fold weaker than that of WT AsfvAP, respectively. These data suggested that the gating residues Tyr81 and Asn273 may play pivotal role in catalytic complex assembly of AsfvAP rather than in DNA binding.

As aforementioned, His145 in the R2 loop forms one water-bridged $\mathrm{H}$-bond interaction with the $5^{\prime}$-phosphate group of dRP (Fig. 3e). Although Arg272 of the R3 region does not interact with $\mathrm{dRP}$, it forms extensive $\mathrm{H}$-bond interactions with the surrounding residues, which may 


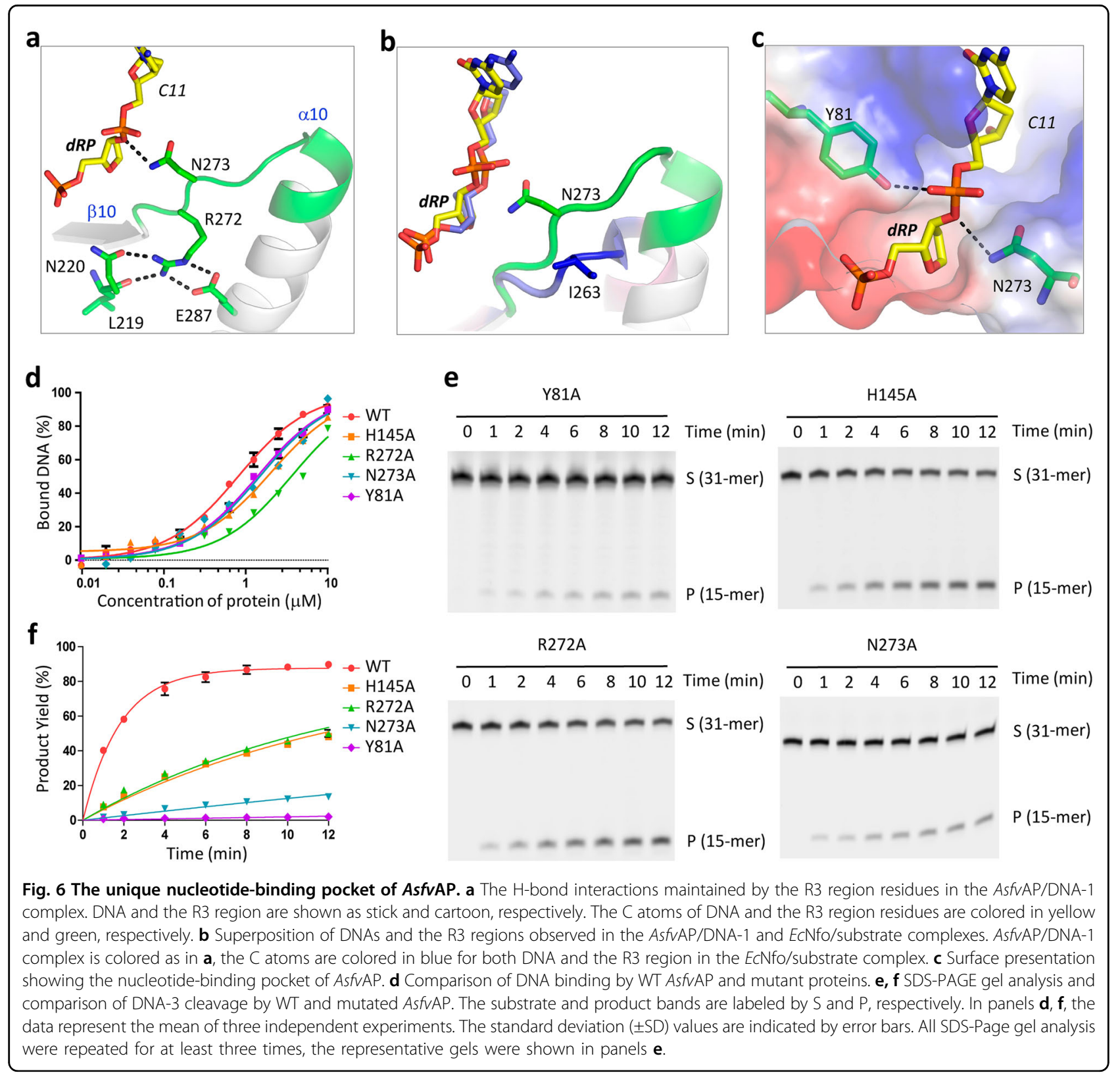

help maintain the proper conformation of Asn273. To investigate the function of His145 and Arg272 of AsfvAP, we constructed two mutants, H145A and R272A. As showed in Fig. 6e, f, the DNA binding affinities of H145A and $\mathrm{R} 272 \mathrm{~A}$ mutants were 2.5 -fold and 4.5 -fold weaker than that of WT AsfvAP, respectively. Although the DNA cleavage activities of the two mutants were comparable to each other, both were 7-fold weaker than that of WT AsfvAP. Hence, our data suggested that His145 and Arg272 are all important for the function of AsfvAP.

\section{AsfvAP has no NIR activity against regular mispairs}

In addition to AP endonuclease activity, previous studies reported that AsfvAP also possessed NIR activity against certain DNAs, such as DNA containing 5,6dihydrothymine (DHT) opposite to adenine ${ }^{24}$. However, probably due to the narrower nucleotide-binding pocket (Fig. 6c), the NIR activity of AsfvAP was significantly weaker than those of $E c \mathrm{Nfo}$ and other class II AP endonucleases. As revealed by our AsfvAP/DNA-2 structure, AsfvAP has no NIR activity against A:G mispair (Fig. 5d). To verify this observation and to investigate whether AsfvAP has NIR activity against other mispairs, we synthesized a set of DNAs (DNA-XY: 5'-GGTAAGGGC AGCGTCCXCGACGAGGAATGCA-FAM-3', 3'-CCAT TCCCGTCGCAGGYGCTGCTCCTTACGT-5', where $X$ and $Y$ denote either $\mathrm{A}, \mathrm{G}, \mathrm{C}$, or $\mathrm{T})$. The in vitro DNA cleavage assays showed that AsfvAP had no NIR activity 
against any regular mispairs embedded in the middle of the substrates (Supplementary Fig. S6a). With the exception of the nucleotides at the $X$ and $Y$ positions, the sequences of DNA- $X Y$ are identical to those of DNA-3 used in the AP endonuclease assay. Interestingly, although no NIR activity was found for DNA-3 (Fig. 1d), AsfvAP exhibited some weak $3^{\prime} \rightarrow 5^{\prime}$ exonuclease activity against DNA- $X Y$ substrates (Supplementary Fig. S6a). Recently, Morera and Ishchenko reported one crystal structure of $E c \mathrm{Nfo}$ (PDB: $4 \mathrm{~K} 1 \mathrm{G})$, which is in complex with dsDNA containing an $\alpha$-anomeric 2 -deoxyadenosine $\cdot T$ base pair $^{30}$. This structure revealed the detailed basis underlying the exonuclease activity of $E c$ Nfo (Supplementary Fig. S6b-c). We believed that AsfvAP may follow the same mechanism in degradation of the terminal nucleotides, since they share the conserved catalytic residues (Figs. 1a and 4d). Rather than substrate-bound or product-bound state, structural superposition suggested that our AsfvAP/DNA-2 complex may represent one product-releasing state.

\section{Discussion}

Although ASFV has caused a substantial loss in agricultural industry in the world over the past century, no vaccine or other useful treatment against this virus has been developed so far. Therefore, it will remain a considerable challenge in the foreseeable future. Better understanding of the structures and unique features of functionally important proteins will be helpful to develop the vaccine and treatment against ASFV. AsfvAP is one key enzyme involved in the BER pathway of ASFV and is essential for ASFV survival in the host cells. In this study, by solving two complex structures of AsfvAP and carrying out binding and cleavage assays, we identified many unique structural features of AsfvAP. And, as revealed by structural comparison, we found that AsfvAP adopts one novel DNA-binding mode. Besides ASFV, class II AP endonucleases are also expressed by many other viruses, such as Acanthamoeba polyphaga mimivirus, Faustovirus ST1, Harvfovirus sp., Kaumoebavirus, Klosneuvirus KNV1, Moumouvirus australiensis, Moumouvirus goulette, Pacmanvirus A23, Satyrvirus sp, and Terrestrivirus sp. Our studies on AsfvAP may also advance our understanding on these viral proteins.

Compared to the homologous proteins, the nucleotidebinding pocket of AsfvAP is much narrower and is more specific for the abasic site. Unlike abasic site, other damaged or modified nucleotides, such as DHT, 5,6dihydrouracil (DHU), and 5-hydroxycytosine $(5 \mathrm{ohC})$, are all poor substrates of AsfvAP. Normally, the nucleobases of these damaged or modified nucleotides will be removed by DNA glycosylase, forming abasic site. AsfvAP cleaves DNA at the $5^{\prime}$ side of the abasic site, generating $3^{\prime}-\mathrm{OH}$ group and $5^{\prime}$-dRP. As supported by the AsfvAP/DNA-1 complex, AsfvAP will not remove dRP from the product strand (Fig. 2b, c). AsfvAP does not recognize and cleave the mispairs embedded in the middle of the DNA duplex (Supplementary Fig. S6a), but it is able to remove the nucleotides (either paired or mispaired) from the $3^{\prime}$ end or nick site of the DNAs in the absence of abasic sites. The gap is then filled by AsfvPolX and sealed by AsfvLIG subsequently. As the fidelities of AsfvPolX and AsfvLIG are very low, they may introduce and tolerate various mispairs at their active sites. Like the AP endonuclease activity, the weak $3^{\prime}$ processing activity of AsfvAP may also play a role in the mutagenesis and genotype formation of ASFV.

ASFV is one of the most complex DNA viruses known to date, and encodes more than 150 proteins. As demonstrated by our present and previous studies, Asf$v$ AP, AsfvPolX and AsfvLIG all possess some unique structural features. Besides these DNA repair proteins, the structures of calcineurin inhibitor protein $\mathrm{A} 238 \mathrm{~L}^{31}$, Bcl-2 like protein $\mathrm{A} 179 \mathrm{~L}^{32}$ and sulfhydryl oxidase $\mathrm{B} 119 \mathrm{~L}^{33}$ of ASFV have also been reported previously. However, the structures of the majority of ASFV proteins are currently unavailable. In the future, it is worth to investigate whether certain ASFV proteins possess unusual features and are capable of cleaning dRP and damaged nucleobases.

Recently, some poly ADP-ribose polymerase (PARP) inhibitors, which can selectively target the DNA repair defect in hereditary breast cancer, have been discovered $^{34}$. The success of the PARP inhibitors has stimulated the development of inhibitors targeting other repair enzymes, such as DNA ligases ${ }^{35-37}$. As reported previously, deletion of AsfvAP coding gene significantly lowered the replication of ASFV, implying that the BER pathway plays a critical role in the life cycle of ASFV. Similar to AsfvPolX and AsfvLIG, AsfvAP also possesses many unique structural features, especially the narrower nucleotide-binding pocket at the active site, and these unique features may serve as an ideal target for designing small molecule inhibitors. Unlike AsfvAP, the AP endonuclease of the pig (SusAPE) belongs to calss I AP endonuclease family, and is closely related $(>90 \%$ nucleotide similarity) with human APE1 (HsAPE1). As revealed by $H s \mathrm{APE} 1^{38}$ and many other homologous protein structures, the overall fold of class I AP endonucleases is very different from that of AsfvAP. Hence, the AsfvAP-specific inhibitors will not interfere with the normal function of SusAPE, but they will impair the ASFV repair process and help combat this deadly virus in the future.

\section{Materials and methods \\ Plasmid construction}

The genes containing the codon-optimized cDNA sequences of WT AsfvAP (Supplementary Table S5) and $E c$ Nfo (Supplementary Table S6) were purchased from 
Shanghai Geneways Biotech Co., Ltd, China. The target fragment was recovered and recombined into pET28Sumo vector. The recombinant vectors (coding for HisSumo-AsfvAP or His-Sumo- $E c N f o$ ) were then transformed into Escherichia coli BL21 DE3 competent cells for protein expression. The recombinant His-Sumo-Asf$v \mathrm{AP}$ coding vector was utilized as template during the plasmid constructions of all AsfvAP mutants, via overlap polymerase chain reactions or site direct mutagenesis according to the manufacturer's protocols. The detailed sequences of the primers are listed in Supplementary Table S5.

\section{Protein expression and purification}

WT and all mutant AsfvAP proteins were expressed and purified using the same procedures. Briefly, the frozen recombinant strains were revived in Lysogeny broth (LB) medium supplemented with $50 \mu \mathrm{g} / \mathrm{mL}$ kanamycin at $37^{\circ} \mathrm{C}$ overnight. Every $20 \mathrm{~mL}$ revived bacterium suspension was inoculated into $1 \mathrm{~L}$ LB medium supplemented with kanamycin $(50 \mu \mathrm{g} / \mathrm{mL})$ and $\mathrm{ZnCl}_{2}(0.2 \mathrm{mM})$, cultured at $37^{\circ} \mathrm{C}$ with continuous shaking. Protein expression was induced at $\mathrm{OD}_{600} \approx 0.6$ by adding of isopropyl $\beta$-D-1thiogalacto-pyranoside (IPTG) at a final concentration of $0.05 \mathrm{mM}$. The induced cultures were then grown at $18^{\circ} \mathrm{C}$ for an additional $18 \mathrm{~h}$.

The cells were harvested by centrifugation, the pellets were resuspended in Buffer A (20 mM MES pH 6.0, $500 \mathrm{mM} \mathrm{NaCl}, 25 \mathrm{mM}$ imidazole) and lysed under high pressure via a cell crusher. The homogenate was clarified by centrifugation and the supernatant was loaded onto a HisTrap ${ }^{\mathrm{TM}}$ HP column equilibrated with Buffer A. The fusion protein was eluted from the column using Buffer B (20 mM MES pH 6.0, $500 \mathrm{mM} \mathrm{NaCl}, 500 \mathrm{mM}$ imidazole) with a gradient. The fractions containing the desired fusion proteins were pooled and dialyzed against Buffer $\mathrm{C}$ (20 mM MES pH 6.0, $500 \mathrm{mM} \mathrm{NaCl}$ ) at $4{ }^{\circ} \mathrm{C}$ for $1 \mathrm{~h}$; Ulp1 protease was added to the sample during the dialysis process. The sample was applied to a HiTrap SP HP column equilibrated with Buffer D (20 mM MES pH 6.0, $100 \mathrm{mM} \mathrm{NaCl}$ ) and eluted using Buffer E (20 mM MES $\mathrm{pH} 6.0,1 \mathrm{M} \mathrm{NaCl}$ ) with a continuous gradient. The target protein was concentrated and loaded onto a Hi 16/60 Superdex G200 column equilibrated with gel filtration buffer (20 mM MES pH 6.0, $200 \mathrm{mM} \mathrm{NaCl).}$

$E c \mathrm{Nfo}$ was expressed using similar procedures as Asf$v$ AP. The cell pellets were harvest and resuspended in Buffer F (20 mM Tris pH 8.0, $500 \mathrm{mM} \mathrm{NaCl}, 25 \mathrm{mM}$ imidazole) and lysed under high pressure. The homogenate was clarified by centrifugation and the supernatant was loaded onto a HisTrap ${ }^{\mathrm{TM}}$ HP column equilibrated with Buffer $F$. The fusion protein was eluted from the column using Buffer G (20 mM Tris pH 8.0, $500 \mathrm{mM}$ $\mathrm{NaCl}, 500 \mathrm{mM}$ imidazole) with a gradient. The fractions containing the desired proteins were pooled and dialyzed against Buffer $\mathrm{F}$ at $4{ }^{\circ} \mathrm{C}$ for $1 \mathrm{~h}$; Ulp1 protease was also added to the sample during the dialysis process. The sample was applied to a HiTrap QP HP column equilibrated with Buffer $\mathrm{H}(20 \mathrm{mM}$ Tris $\mathrm{pH} 8.0,100 \mathrm{mM} \mathrm{NaCl})$ and eluted using Buffer $\mathrm{E}$ with a continuous gradient.

\section{In vitro DNA binding and cleavage assays}

All DNAs used in the binding and cleavage assays were purchased from Shanghai Generay Biotech Co., Ltd. The DNA substrates were assembled in a molar ratio of 1:1 in reaction buffer (20 mM MES pH $5.0-7.0$ or $20 \mathrm{mM}$ Tris $\mathrm{pH} 7.5-8.0,200 \mathrm{mM} \mathrm{NaCl}, 5 \mathrm{mM} \mathrm{MgCl} 2,0.05 \% \mathrm{NP} 40,5 \%$ Glycerol). For cleavage assays, a $50-\mu \mathrm{L}$ reaction system (composed of $40 \mu \mathrm{L}$ gel filtration buffer, $5 \mu \mathrm{L} 2 \mu \mathrm{M}$ DNA, and $5 \mu \mathrm{L}$ protein) was established. The final protein concentrations are $0.025 \mu \mathrm{M}$. The reactions $(5 \mu \mathrm{L})$ were carried out at $37^{\circ} \mathrm{C}$ and quenched by adding of $5 \mu \mathrm{L}$ termination buffer (90\% formamide, 20 mM EDTA, 0.05\% bromophenol blue, and $0.05 \%$ xylene blue) at various time points. Eight $\mu \mathrm{L}$ samples were loaded onto pre-warmed $18 \%$ urea sequencing gels and run at $18-20 \mathrm{~W}$ and $40-45^{\circ} \mathrm{C}$ for $60 \mathrm{~min}$. The gel was visualized using Typhoon FLA 9000, and intensities of the substrate and product bonds were quantified by ImageQuantTL. Data were then fitted to the exponential $Y=Y_{\max }\left[1-e^{\left(-K_{\mathrm{obs}} t\right)}\right]$ using non-linear regression in GraphPad Prism 5. The observed rate constant $\left(K_{\text {obs }}\right)$ and maximum cleavage yield $\left(Y_{\max }\right)$ were determined from the regression curve (Supplementary Tables S2-4).

Substrates used in fluorescence polarization were diluted to $20 \mathrm{nM}$ with reaction buffer. A $200-\mu \mathrm{L}$ reaction system, composed of $100 \mu \mathrm{L}$ DNA $(20 \mathrm{nM})$ and $100 \mu \mathrm{L}$ proteins was established. The final protein concentration was fixed at $10 \mathrm{nM}$. The samples were incubated at $0{ }^{\circ} \mathrm{C}$ for $30 \mathrm{~min}$. All FP measurements were performed at room temperature in the dark using Synergy 2 Muti-Mode Microplate Reader (BioTek). The data were fitted to the exponential $Y=$ Bottom $+($ Top-Bottom $) /\left(1+10^{(\operatorname{LogEC} 50-X)}\right)$ using nonlinear regression in GraphPad Prism. The $K_{\mathrm{d}}$ values (Supplementary Table S4) were determined from the regression curve. All the experiments were repeat for at least three times.

\section{Crystallization and data collection}

All DNAs used in the structural studies were dissolved in $\mathrm{ddH}_{2} \mathrm{O}$. The crystallization samples were prepared by mixing AsfvAP and DNA-1 or DNA-2 together at room temperature. The final concentration of the protein is $0.2 \mathrm{mM}$, and the concentration of DNA-1 or DNA-2 duplexes is $0.22 \mathrm{mM}$ for the crystallization attempt. The initial crystallization conditions were identified at $18^{\circ} \mathrm{C}$ using the crystallization robot system and commercial crystallization kits. During initial screening, the sitting-drop 
vapor diffusion method was used, whereas all the crystal optimization procedures were performed using the hanging-drop vapor diffusion method. The AsfvAP/DNA1 crystals were grown under the condition composed of $0.1 \mathrm{M}$ BIS-TRIS $\mathrm{pH} 5.5,13 \% \mathrm{w} / \mathrm{v}$ PEG 10,000 and $0.1 \mathrm{M}$ ammonium acetate. The crystallization condition of AsfvAP/DNA-2 is composed of 16\% w/v PEG 3350 and $0.1 \mathrm{M}$ potassium sodium tartrate tetrahydrate.

Both AsfvAP/DNA-1 and AsfvAP/DNA-2 crystals were cryoprotected using their mother liquor supplemented with $25 \%$ glycerol and snap-frozen in liquid nitrogen. Xray diffraction data were collected on beamline BL17U1, BL18U1, and BL19U1 at the Shanghai Synchrotron Radiation Facility (SSRF). Data processing was carried out using the HKL2000 or HKL3000 programs ${ }^{39}$. The data collection and processing statistics are summarized in Supplementary Table S1.

\section{Structure determination and refinement}

The AsfvAP/DNA-1 structure was solved by singlewavelength anomalous diffraction (SAD) method ${ }^{40}$ using the anomalous signal of $\mathrm{Zn}^{2+}$ cofactor ions, which were co-purified with AsfvAP protein. The AutoSol program ${ }^{41}$ embedded in the Phenix suite ${ }^{42}$ was utilized to determine the structure, which identified all the six $\mathrm{Zn}^{2+}$ ions within the asymmetric unit. The phasing figure-of-merit (FOM) value is 0.35 . The initial model, which covers approximately $60 \%$ of protein residues in the asymmetric unit, was built using the Autobuild program and was refined against the diffraction data using the Refmac5 program ${ }^{43}$ of CCP $4 \mathrm{i}^{44}$. During refinement, $5 \%$ of randomly selected data was set aside for free $\mathrm{R}$-factor cross validation calculations. The $2 \mathrm{~F}_{\mathrm{o}}-\mathrm{F}_{\mathrm{c}}$ and $\mathrm{F}_{\mathrm{o}}-\mathrm{F}_{\mathrm{c}}$ electron density maps were regularly calculated and used as guides for building the missing amino acids, DNA, and solvent molecules using $\mathrm{COOT}^{45}$. The AsfvAP/DNA-2 structure was solved using the molecular replacement (MR) method with the Phaser program of the CCP4i suite; the AsfvAP molecule of the AsfvAP/DNA-1 structure was used as the search model. The final refinement of all structures was done using the phenix.refine program ${ }^{46}$ of Phenix. The structural refinement statistics are summarized in Supplementary Table S1.

\section{Accession codes}

Structural factors and coordinates have been deposited in the Protein Data Bank under accession codes 6KI3 and 6KHY for the AsfvAP/DNA-1 and AsfvAP/DNA-2 complex structures, respectively.

\section{Acknowledgements}

We thank the staff of BL17U1 beamline at the Shanghai Synchrotron Radiation Facility (SSRF), the staff of BL18U1, BL19U1 beamlines of National Faciltiy for Protein Science Shanghai for help during data collection. This work was supported by the National Natural Science Foundation of China (31870721), the Special Foundation of Science and Technology Resources Survey from Ministry of Science and Technology (SQ2019FY010009), the Key Research and Development Project of China (2016YFA0500600), and Open Funding Project of the State Key Laboratory of Bioreactor Engineering.

\section{Author details}

${ }^{1}$ State Key Laboratory of Genetic Engineering, Collaborative Innovation Center of Genetics and Development, Shanghai Public Health Clinical Center, School of Life Sciences, Fudan University, 200438 Shanghai, China. ${ }^{2}$ State Key Laboratory of Bioreactor Engineering, East China University of Science and Technology, 200237 Shanghai, China. ${ }^{3}$ Shanghai Public Health Clinical Center, School of Life Sciences, Fudan University, 200438 Shanghai, China. ${ }^{4}$ State Key Laboratory for Infectious Disease Prevention and Control, Collaborative Innovation Center for Diagnosis and Treatment of Infectious Diseases, National Institute for Communicable Disease Control and Prevention, Chinese Center for Disease Control and Prevention, Changping, 102206 Beijing, China.

${ }^{5}$ National Center for Protein Science Shanghai, Shanghai Advanced Research Institute, Chinese Academy of Sciences, 201210 Shanghai, China

\section{Author contributions}

Y.Q.C., X.C., and Q.H. produced and purified the proteins, and performed the biochemical assays. Y.Q.C., X.C., Z.W.S., Y.Q.G., Y.Y.L., and Y.C. grow the crystals and collected the X-ray data. Y.Q.C., Y.J.G., and J.H.G. determined the structures. Y.Q.C., X.C., H.H.L., Q.Y.W., J.X.L., J.B.M., Y.Z.Z., Y.J.G., and J.H.G. analyzed the data. Y.Q.C., Y.Z.Z., Y.J.G., and J.H.G. wrote the paper.

Conflict of interest

The authors declare that they have no conflict of interest.

\section{Publisher's note}

Springer Nature remains neutral with regard to jurisdictional claims in published maps and institutional affiliations.

Supplementary Information accompanies the paper at (https://doi.org/ 10.1038/s41421-020-0146-2).

Received: 1 November 2019 Accepted: 13 February 2020

Published online: 17 March 2020

\section{References}

1. Tulman, E. R., Delhon, G. A., Ku, B. K. \& Rock, D. L. African swine fever virus. Curr. Top. Microbiol. Immunol. 328, 43-87 (2009).

2. Vinuela, E. African swine fever virus. Curr. Top. Microbiol. Immunol. 116, 151-170 (1985)

3. Gogin, A., Gerasimov, V., Malogolovkin, A. \& Kolbasov, D. African swine fever in the North Caucasus region and the Russian Federation in years 2007-2012. Virus Res. 173, 198-203 (2013).

4. Costard, S., Mur, L., Lubroth, J., Sanchez-Vizcaino, J. M. \& Pfeiffer, D. U. Epidemiology of African swine fever virus. Virus Res. 173, 191-197 (2013).

5. Zhou, X. et al. Emergence of African Swine Fever in China, 2018. Transbound. Emerg. Dis. 65, 1482-1484 (2018).

6. Sanchez-Vizcaino, J. M., Mur, L. \& Martinez-Lopez, B. African swine fever: an epidemiological update. Transbound. Emerg. Dis. 59(Suppl 1), 27-35 (2012).

7. Yanez, R. J. et al. Analysis of the complete nucleotide sequence of African swine fever virus. Virology 208, 249-278 (1995).

8. Alcami, A., Carrascosa, A. L. \& Vinuela, E. Interaction of African swine fever virus with macrophages. Virus Res. 17, 93-104 (1990).

9. Akaike, T. Role of free radicals in viral pathogenesis and mutation. Rev. Med. Virol. 11, 87-101 (2001).

10. Forman, H. J. \& Torres, M. Redox signaling in macrophages. Mol. Asp. Med. 22, 189-216 (2001).

11. Showalter, A. K. \& Tsai, M. D. A DNA polymerase with specificity for five base pairs. J. Am. Chem. Soc. 123, 1776-1777 (2001).

12. Lamarche, B. J., Showalter, A. K. \& Tsai, M. D. An error-prone viral DNA ligase. Biochemistry 44, 8408-8417 (2005).

13. Boshoff, C. I., Bastos, A. D., Gerber, L. J. \& Vosloo, W. Genetic characterisation of African swine fever viruses from outbreaks in southern Africa (1973-1999). Vet. Microbiol. 121, 45-55 (2007). 
14. Bastos, A. D. et al. Genotyping field strains of African swine fever virus by partial p72 gene characterisation. Arch. Virol. 148, 693-706 (2003).

15. Achenbach, J. E. et al. Identification of a new genotype of African swine fever virus in domestic pigs from Ethiopia. Transbound. Emerg. Dis. 64, 1393-1404 (2017).

16. Redrejo-Rodriguez, M., Rodriguez, J. M., Suarez, C., Salas, J. \& Salas, M. L. Involvement of the reparative DNA polymerase Pol $X$ of African swine fever virus in the maintenance of viral genome stability in vivo. J. Virol. 87, 9780-9787 (2013).

17. Redrejo-Rodriguez, M., Garcia-Escudero, R., Yanez-Munoz, R. J., Salas, M. L. \& Salas, J. African swine fever virus protein pE296R is a DNA repair apurinic/ apyrimidinic endonuclease required for virus growth in swine macrophages. J. Virol. 80, 4847-4857 (2006)

18. Wu, W. J. et al. How a low-fidelity DNA polymerase chooses non-Watson-Crick from Watson-Crick incorporation. J. Am. Chem. Soc. 136, 4927-4937 (2014).

19. Maciejewski, M. W. et al. Solution structure of a viral DNA repair polymerase. Nat. Struct. Biol. 8, 936-941 (2001).

20. Showalter, A. K., Byeon, I. J., Su, M. I. \& Tsai, M. D. Solution structure of a viral DNA polymerase $X$ and evidence for a mutagenic function. Nat. Struct. Biol. 8 , 942-946 (2001)

21. Chen, $Y$. et al. Unique $5^{\prime}-P$ recognition and basis for $\mathrm{dG}$ :dGTP misincorporation of ASFV DNA polymerase X. PLOS Biol. 15, e1002599 (2017).

22. Chen, $Y$. et al. Structure of the error-prone DNA ligase of African swine fever virus identifies critical active site residues. Nat. Commun. 10, 387 (2019).

23. Lindahl, T. \& Wood, R. D. Quality control by DNA repair. Science $\mathbf{2 8 6}$ 1897-1905 (1999)

24. Lamarche, B. J. \& Tsai, M. D. Contributions of an endonuclease IV homologue to DNA repair in the African swine fever virus. Biochemistry 45, 2790-2803 (2006).

25. Redrejo-Rodriguez, M., Ishchenko, A. A., Saparbaev, M. K., Salas, M. L. \& Salas, J. African swine fever virus AP endonuclease is a redox-sensitive enzyme that repairs alkylating and oxidative damage to DNA. Virology 390, 102-109 (2009).

26. Canton, J., Khezri, R., Glogauer, M. \& Grinstein, S. Contrasting phagosome pH regulation and maturation in human $\mathrm{M} 1$ and $\mathrm{M} 2$ macrophages. Mol. Biol. Cell 25, 3330-3341 (2014).

27. Zhang, W. et al. Crystal structure of the apurinic/apyrimidinic endonuclease IV from Mycobacterium tuberculosis. Biochem. Biophys. Res. Commun. 498, 111-118 (2018)

28. Asano, R. et al. An additional C-terminal loop in endonuclease IV, an apurinic/ apyrimidinic endonuclease, controls binding affinity to DNA. Acta Crystallogr. D. Biol. Crystallogr. 67, 149-155 (2011).

29. Garcin, E. D. et al. DNA apurinic-apyrimidinic site binding and excision by endonuclease IV. Nat. Struct. Mol. Biol. 15, 515-522 (2008).
30. Mazouzi, A. et al. Insight into mechanisms of $3^{\prime}-5^{\prime}$ exonuclease activity and removal of bulky 8,5'-cyclopurine adducts by apurinic/apyrimidinic endonucleases. Proc. Natl Acad. Sci. USA 110, E3071-E3080 (2013).

31. Grigoriu, S. et al. The molecular mechanism of substrate engagement and immunosuppressant inhibition of calcineurin. PLoS Biol. 11, e1001492 (2013).

32. Banjara, S., Caria, S., Dixon, L. K., Hinds, M. G. \& Kvansakul, M. Structural Insight into African swine fever virus A179L-Mediated Inhibition of Apoptosis. J. Virol. 91, e02228-16 (2017)

33. Hakim, M. \& Fass, D. Dimer interface migration in a viral sulfhydryl oxidase. J. Mol. Biol. 391, 758-768 (2009).

34. Lord, C. J. \& Ashworth, A. Targeted therapy for cancer using PARP inhibitors. Curr. Opin. Pharmacol. 8, 363-369 (2008).

35. Tseng, H. M. et al. A high-throughput scintillation proximity-based assay for human DNA ligase IV. Assay. Drug Dev. Technol. 10, 235-249 (2012).

36. Sun, D. \& Urrabaz, R. Development of non-electrophoretic assay method for DNA ligases and its application to screening of chemical inhibitors of DNA ligase I. J. Biochem. Biophys. Methods 59, 49-59 (2004).

37. Zhong, S. et al. Identification and validation of human DNA ligase inhibitors using computer-aided drug design. J. Med. Chem. 51, 4553-4562 (2008).

38. Mol, C. D., Izumi, T., Mitra, S. \& Tainer, J. A. DNA-bound structures and mutants reveal abasic DNA binding by APE1 and DNA repair coordination [corrected] Nature 403, 451-456, https://doi.org/10.1038/35000249 (2000).

39. Minor, W., Cymborowski, M., Otwinowski, Z. \& ChruszCZ, M. HKL-3000: the integration of data reduction and structure solution-from diffraction images to an initial model in minutes. Acta Crystallogr. D. Biol. Crystallogr. 62, 859-866 (2006).

40. Giacovazzo, C. \& Siliqi, D. Phasing via SAD/MAD data: the method of the joint probability distribution functions. Acta Crystallogr. D. Biol. Crystallogr. 60, 73-82 (2004).

41. Terwilliger, T. C. et al. Decision-making in structure solution using Bayesian estimates of map quality: the PHENIX AutoSol wizard. Acta Crystallogr. D. Biol. Crystallogr. 65, 582-601 (2009).

42. Adams, P. D. et al. PHENIX: building new software for automated crystallographic structure determination. Acta Crystallogr. D. Biol. Crystallogr. 58, 1948-1954 (2002).

43. Murshudov, G. N. et al. REFMAC5 for the refinement of macromolecular crystal structures. Acta Crystallogr. D. Biol. Crystallogr. 67, 355-367 (2011).

44. Potterton, E., Briggs, P., Turkenburg, M. \& Dodson, E. A graphical user interface to the CCP4 program suite. Acta Crystallogr. D. Biol. Crystallogr. 59, 1131-1137 (2003).

45. Emsley, P. \& Cowtan, K. Coot: model-building tools for molecular graphics. Acta Crystallogr. D. Biol. Crystallogr. 60, 2126-2132 (2004).

46. Afonine, P. V. et al. Towards automated crystallographic structure refinement with phenix.refine. Acta Crystallogr. D. Biol. Crystallogr. 68, 352-367 (2012). 